\title{
Controlling qubit arrays with anisotropic $X X Z$ Heisenberg interaction by acting on a single qubit
}

\author{
Rahel Heule ${ }^{1}$, C. Bruder ${ }^{1}$, Daniel Burgarth ${ }^{2,3}$, and Vladimir M. Stojanović ${ }^{1}$ \\ 1 Department of Physics, University of Basel, Klingelbergstrasse 82, CH-4056 Basel, Switzerland \\ 2 Institute for Mathematical Sciences, Imperial College London, SW7 2PG, United Kingdom \\ ${ }^{3}$ QOLS, The Blackett Laboratory, Imperial College London, Prince Consort Road, SW7 2BW, United Kingdom
}

Received: date / Revised version: date

\begin{abstract}
We investigate anisotropic $X X Z$ Heisenberg spin-1/2 chains with control fields acting on one of the end spins, with the aim of exploring local quantum control in arrays of interacting qubits. In this work, which uses a recent Lie-algebraic result on the local controllability of spin chains with "alwayson" interactions, we determine piecewise-constant control pulses corresponding to optimal fidelities for quantum gates such as spin-flip (NOT), controlled-NOT (CNOT), and square-root-of-SWAP $(\sqrt{\mathrm{SWAP}})$. We find the minimal times for realizing different gates depending on the anisotropy parameter $\Delta$ of the model, showing that the shortest among these gate times are achieved for particular values of $\Delta$ larger than unity. To study the influence of possible imperfections in anticipated experimental realizations of qubit arrays, we analyze the robustness of the obtained results for the gate fidelities to random variations in the control-field amplitudes and finite rise time of the pulses. Finally, we discuss the implications of our study for superconducting charge-qubit arrays.
\end{abstract}

PACS. 03.67.Ac Quantum algorithms and protocols - 03.67.Lx Quantum computation

\section{Introduction}

Coherent control of quantum systems is one of the prerequisites for quantum information processing. While already simple arguments lead to the conclusion that almost any coupled quantum system can be controlled in principle [1, the mathematical foundations of the subject are based on the notion of controllability and formulated using the language of Lie algebras 2. In particular, a system is completely controllable if its internal dynamics governed by external fields can give rise to an arbitrary unitary transformation in the Hilbert space of the system [3]. Both state control and the more general operator control have been implemented in a variety of systems 4 .

Recent quantum control studies have focused their attention on interacting systems. A familiar example is furnished by spin chains, systems that can be used as data buses [5 for state- 6, 7, 8, 9 and entanglement transfer [10. In such systems, "always-on" interactions between the constituents (typically nearest neighbors) allow for a global control of the system dynamics by manipulating only a small subsystem, in the extreme case a single spin. The main question is then what is the smallest possible subsystem of a given system that one needs to act upon to ensure the complete controllability, or, at least, the ability to perform certain pre-determined unitary transformations. This is the central idea behind the local-control approach.
The fact that the local-control approach can be advantageous in interacting systems provides an incentive for identifying minimal controlling resources that guarantee controllability in particular classes of systems. Quite recently, several Lie-algebraic results pertaining to local control of spin chains have been obtained 11, 12, 13, 14, 15. For example, it was demonstrated that acting only on one of the end spins of an $X X Z$-Heisenberg spin chain ensures complete controllability of the chain [12. Adopting the last result as our point of departure, in this paper we investigate the feasibility of local operator control in qubit arrays modeled as spin- $1 / 2$ chains with Heisenberg interaction. In contrast to our recent proof-of-principle study [16], where only the isotropic Heisenberg-coupling case was addressed, in the present work we discuss the case of (anisotropic) $X X Z$ coupling. The main motivation stems from the relevance of the $X X Z$-case for implementations of Josephson-junction based superconducting qubit arrays 17, 18, 19.

We determine piecewise-constant control fields, acting only on the first spin in the chain, which lead to the highest possible fidelities for a selected set of quantum logic operations: the spin-flip (NOT) of the last spin in the chain, as well as the controlled-NOT (CNOT) and the square-rootof-SWAP $(\sqrt{\text { SWAP }})$ gates applied to the last two spins. We optimize the gate fidelities with respect to the controlfield amplitudes for three-spin chains. We then carry out a 
sensitivity analysis, i.e., discuss the robustness of the obtained results with respect to random errors in the control fields, as well as finite rise/decay-times for control-field amplitudes. The present work is concerned with $X X Z$ Heisenberg spin chains and our conclusions apply to any physical realization of qubit arrays with this type of coupling [17, 18].

\section{System and method}

The total Hamiltonian of a Heisenberg spin- $1 / 2$ chain of length $N_{s}$ reads

$$
H(t)=H_{0}+H_{c}(t)
$$

where

$$
H_{0}=J \sum_{i=1}^{N_{s}-1}\left(S_{i, x} S_{i+1, x}+S_{i, y} S_{i+1, y}+\Delta S_{i, z} S_{i+1, z}\right)
$$

is a $X X Z$ Heisenberg part with anisotropy $\Delta$, and

$$
H_{c}(t)=h_{x}(t) S_{1 x}+h_{y}(t) S_{1 y}
$$

a Zeeman-like control part, with control fields $h_{x}(t), h_{y}(t)$ acting only on the first spin. In what follows, we will also employ control Hamiltonians with fields in the $x$ and $z$ directions. Whether the $X X Z$ spin chain under consideration is ferromagnetic or antiferromagnetic is not crucial here, as we are concerned with operator control; aspects such as, for example, the different nature of the ground states in the two cases (separable vs. entangled) would only be consequential for issues related to, e.g., state control or entanglement transfer. For definiteness, we will assume that $J>0$ and $\Delta>0$. It is useful to recall that the one-dimensional $X X Z$ model has an antiferromagnetic ground state for $\Delta \geq 1$, a ferromagnetic one for $\Delta<-1$, while for the intermediate values of $\Delta$ it is characterized by a critical gapless (quasi-long-range ordered) phase [20].

For convenience, we hereafter set $\hbar=1$ and, in addition, express all frequencies and control fields in units of the coupling strength $J$. Consequently, all times in the problem are expressed in units of $1 / J$.

Since implementing control fields with a complex time dependence is difficult, we resort to piecewise-constant ones according to the following scheme. At $t=0$ we start acting on the first spin of the chain with an $x$ control pulse of amplitude $h_{x, 1}$, which is kept constant until $t=T$. Thus the system is governed by the Hamiltonian $H_{x, 1} \equiv H_{0}+h_{x, 1} S_{1 x}$. We then apply a $y$ pulse with the amplitude $h_{y, 1}$ (Hamiltonian $H_{y, 1} \equiv H_{0}+h_{y, 1} S_{1 y}$ ) over the next interval of length $T$, etc. This sequence repeats until $N_{t}$ pulses are carried out at $t=t_{f} \equiv N_{t} T$. The full time evolution is described by

$$
U\left(t_{f}\right)=U_{y, N_{t} / 2} U_{x, N_{t} / 2} \ldots U_{y, 1} U_{x, 1}
$$

where $U_{x, i} \equiv e^{-i H_{x, i} T}$ and $U_{y, i} \equiv e^{-i H_{y, i} T}$ are the respective time-evolution operators corresponding to $H_{x, i}$ and $H_{y, i}$, which can be evaluated using their spectral form.
Our control objectives (target unitary operations) are both one-qubit gates, such as the spin-flip (NOT) operation on the last spin of the chain $X_{N_{s}}:=\mathbb{1} \otimes \mathbb{1} \otimes \ldots \otimes \mathbb{1} \otimes X$ ( $X$ being the Pauli matrix), and some entangling twoqubit gates. For instance, $\mathrm{CNOT}_{N_{s}}:=\mathbb{1} \otimes \mathbb{1} \otimes \ldots \otimes$ $\mathbb{1} \otimes$ CNOT performs the controlled-NOT operation on the last two qubits in the chain. Similarly, $\sqrt{\mathrm{SWAP}}_{N_{s}}:=$ $\mathbb{1} \otimes \mathbb{1} \otimes \ldots \otimes \mathbb{1} \otimes \sqrt{\text { SWAP }}$ performs the $\sqrt{\text { SWAP }}$ operation on the same pair of qubits.

Unlike in many other control studies [11, which make use of single-excitation subspaces, we retain the full Hilbert space of the system. This puts constraints on the system size that can be treated within our framework. In what follows, we discuss three-spin chains.

\section{Controllability and reachability}

In Ref. [12 a very general graph-infection criterion was proven, which - as a special case - guarantees the complete controllability of $X X Z$ Heisenberg spin chains through acting on one end spin. The more conventional approaches for proving complete controllability entail finding the dimension of the relevant dynamical Lie algebra, a task for which special algorithms have been developed [21. In the present problem, such an algebra is generated by the skewHermitian traceless operators $\left\{-i H_{0},-i S_{1 x},-i S_{1 y}\right\}$ and has dimension $d^{2}-1$, where $d \equiv 2^{N_{s}}$ is the dimension of the Hilbert space of the system. Being generated by traceless operators, this algebra is then isomorphic to $s u(d)$, the Lie algebra associated with the special unitary group $S U(d)$ 22].

Setting aside the issue of complete controllability, one might be interested to know if some particular unitary operations - on an otherwise not completely controllable system [23 24] - are possible with an even smaller degree of manipulation, e.g.., a control field only in one direction. For such operations, equation (44) goes over into $U\left(t_{f}\right)=$ $U_{x, N_{t}} \ldots U_{x, 1}$. For example, the $X_{N_{s}}$ and $\sqrt{\mathrm{SWAP}_{N_{s}}}$ gates require only a control field in the $x$ direction. To demonstrate this for $X_{N_{s}}$, let $\mathcal{L}_{x}$ be the dynamical Lie algebra generated by $-i H_{0}$ and $-i S_{1 x}$, a subalgebra of $s u(d)$ with dimension 30 in a three-spin $X X Z$ chain (note that the counterpart of this algebra in the isotropic-coupling case has smaller dimension, namely 18). For showing that $X_{N_{s}}$ belongs to the connected Lie subgroup $e^{\mathcal{L}_{x}}$ of $S U(d)$ it suffices to find an element $A \in \mathcal{L}_{x}$ such that $X_{N_{s}}=e^{A}$. Using the repeated commutators of the generators of $\mathcal{L}_{x}$, it can be demonstrated that $X_{N_{s}}$ is an element of this algebra. $X_{N_{s}}$ is both unitary and Hermitian, implying that $X_{N_{s}}^{2}=\mathbb{1}$. It is then easy to show that $A=-i \frac{\pi}{2} X_{N_{s}}$, an element of $\mathcal{L}_{x}$, fulfills $e^{A}=-i X_{N_{s}}$. Therefore, $X_{N_{s}}$ is reachable using only an $x$ control field. Recalling that the $\sqrt{\text { SWAP }}$ gate on two qubits is given by 25 ]

$$
\sqrt{\mathrm{SWAP}}=e^{i \frac{\pi}{8}} e^{-i \frac{\pi}{8}(X \otimes X+Y \otimes Y+Z \otimes Z)},
$$

the reachability of $\sqrt{\mathrm{SWAP}}_{N_{s}}$ using an $x$ control only readily follows from the fact that $\mathbb{1} \otimes \mathbb{1} \otimes \ldots \otimes \mathbb{1} \otimes(X \otimes$ $X+Y \otimes Y+Z \otimes Z)$ is an element of $\mathcal{L}_{x}$. 

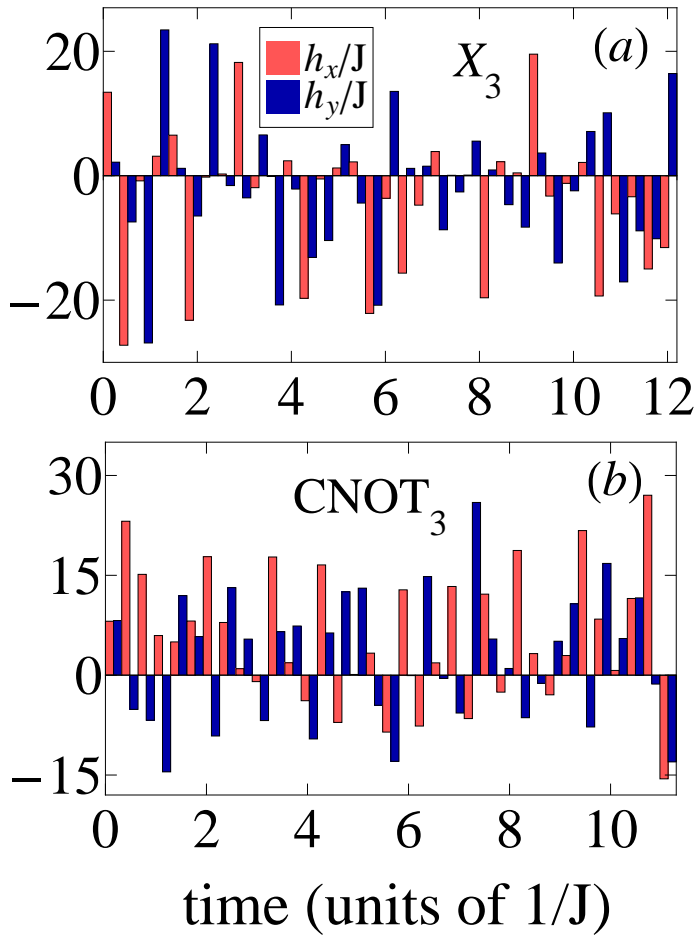

Fig. 1. Optimal control sequences for $\Delta=5$ realizing the (a) $X_{3}$ and (b) $\mathrm{CNOT}_{3}$ gates with fidelity higher than 0.999 .

\section{Target gates and minimal gate times}

In this section our goal is to find control fields leading to optimal fidelities for a chosen set of quantum gates, with a particular emphasis on minimal times needed for realizing different gates depending on the anisotropy $\Delta$.

In quantum operator control, the figure of merit is the gate fidelity

$$
F\left(t_{f}\right)=\frac{1}{d}\left|\operatorname{tr}\left[U^{\dagger}\left(t_{f}\right) U_{\text {target }}\right]\right|,
$$

where $U\left(t_{f}\right)$ is the time-evolution operator of the system at time $t=t_{f}$ (Eq. (4) ) and $U_{\text {target }}$ stands for the quantum gate that we want to realize. We perform optimization, i.e., maximize the gate fidelity with respect to the $N_{t}$ control-field amplitudes, for varying number $\left(N_{t}\right)$ and durations $(T)$ of pulses (hence different total evolution times $\left.t_{f}\right)$. We make use of a quasi-Newton method due to Broyden, Fletcher, Goldfarb, and Shanno (BFGSalgorithm) 26. It should be stressed that, much like other optimization approaches, this algorithm ensures only convergence to a local maximum. Therefore, to determine a globally-optimal sequence of control-field amplitudes (for a given target gate and given value of $\Delta$ ) we ought to repeat the optimization process for a number of different initial guesses for these amplitudes. We generate these initial guesses using a uniform random number generator [26.

An alternative to fixing the pulse durations and maximizing over the control-field amplitudes would be to keep the amplitudes constant and treat the pulse durations as variable control parameters. However, we choose optimization over the control-field amplitudes since this approach
Table 1. Minimal times (in units of $J^{-1}$ ) needed to reach fidelities higher than 0.999 for the relevant gates in the $x-y$ control case. The corresponding values in the $x-z$ control case are given in the brackets.

\begin{tabular}{rrrrrrr}
\hline \multicolumn{1}{c}{$\Delta$} & \multicolumn{2}{c}{$\mathrm{X}_{3}$} & \multicolumn{2}{c}{$\mathrm{CNOT}_{3}$} & \multicolumn{2}{c}{$\sqrt{\mathrm{SWAP}_{3}}$} \\
\hline 0.1 & 23.3 & $(19.0)$ & 73.2 & $(60.2)$ & 14.2 & $(14.2)$ \\
0.2 & 23.9 & $(23.8)$ & 33.5 & $(28.7)$ & 20.7 & $(21.2)$ \\
0.7 & 18.2 & $(18.0)$ & 22.2 & $(22.6)$ & 18.9 & $(16.8)$ \\
0.8 & 16.5 & $(16.9)$ & 22.8 & $(21.6)$ & 16.6 & $(16.9)$ \\
0.9 & 15.4 & $(13.9)$ & 18.4 & $(18.2)$ & 1.6 & $(1.6)$ \\
1.0 & 15.0 & $(14.9)$ & 17.3 & $(16.7)$ & 1.5 & $(1.5)$ \\
1.1 & 15.4 & $(15.8)$ & 17.3 & $(16.4)$ & 1.5 & $(1.5)$ \\
1.2 & 14.8 & $(14.8)$ & 20.6 & $(16.7)$ & 14.9 & $(12.2)$ \\
1.3 & 16.2 & $(16.0)$ & 20.0 & $(19.0)$ & 15.2 & $(14.8)$ \\
2.0 & 15.8 & $(15.1)$ & 12.3 & $(12.4)$ & 12.8 & $(9.9)$ \\
3.0 & 12.0 & $(13.4)$ & 12.0 & $(12.2)$ & 10.2 & $(8.8)$ \\
4.0 & 12.2 & $(13.0)$ & 12.2 & $(12.2)$ & 8.0 & $(6.6)$ \\
5.0 & 12.2 & $(13.2)$ & 11.2 & $(11.1)$ & 5.4 & $(5.3)$ \\
6.0 & 12.0 & $(13.5)$ & 11.5 & $(11.3)$ & 4.4 & $(4.4)$ \\
7.0 & 12.9 & $(13.9)$ & 11.8 & $(9.8)$ & 3.8 & $(2.0)$ \\
8.0 & 12.9 & $(14.5)$ & 13.3 & $(11.7)$ & 3.3 & $(3.3)$ \\
9.0 & 13.6 & $(15.0)$ & 13.1 & $(10.4)$ & 4.3 & $(3.0)$ \\
10.0 & 14.8 & $(16.6)$ & 14.4 & $(11.9)$ & 3.9 & $(3.9)$ \\
11.0 & 21.7 & $(17.9)$ & 13.1 & $(10.8)$ & 2.4 & $(2.4)$ \\
12.0 & 22.1 & $(20.4)$ & 19.3 & $(12.0)$ & 2.2 & $(3.3)$ \\
\hline
\end{tabular}

allows us to easily fix $t_{f}$ and determine its minimal value for implementing the desired gate for any fixed value of the parameter $\Delta$.

The obtained results for the gate fidelities have the following two salient features. Firstly, for fixed parameters of the model and fixed total evolution time $t_{f}$, the fidelity for any given gate can increase significantly with increasing $N_{t}$ (or, equivalently, decreasing $T$ ). In other words, more rapid switching leads to higher fidelities. For instance, in the case of the $\mathrm{CNOT}_{3}$ gate with $\Delta=1.3$ and $t_{f}=30$, for $N_{t}=10,20,30,40,50,60,70$ we obtain the respective fidelities $F=0.455,0.697,0.837,0.953,0.995,1-10^{-4}, 1-$ $10^{-8}$. Secondly, for each gate there exists a minimal value of $t_{f}$ (i.e., minimal gate time), below which fidelities close to unity cannot be reached regardless of the value of $N_{t}$. The obtained minimal gate times for different values of $\Delta$ in the $x-y(x-z)$ control cases are given in Table 11. Apparently, there exists an optimal value of $\Delta$ which cor- 
responds to the shortest among these times. For the $X_{3}$ and $\mathrm{CNOT}_{3}$ gates, for example, these values are around $\Delta=5$. The corresponding optimal sequences of $x$ and $y$ control pulses for the $X_{3}$ and $\mathrm{CNOT}_{3}$ gates are shown in Figures 1(a) and 1(b), respectively. Since ideal steplike pulses cannot be realized in practice, in Ref. [16] we also studied frequency-filtered control fields and showed that sufficiently high fidelities can still be retained.

In Table 2 the minimal times are given for the $X_{3}$ and $\sqrt{\mathrm{SWAP}}_{3}$ gates realized using only an $x$ control field. It is interesting to compare these minimal times to the above case with both $x$ and $y$ (or $x$ and $z$ ) controls. For small values of $\Delta$ (with the exception of $\Delta=1$ ) the minimal times for realizing the $X_{3}$ gate in the $x$-only control case are significantly longer than their counterparts in the $x-y$ $(x-z)$ case. In contrast, for larger $\Delta$ these times become more and more similar. Finally, for $\Delta \geq 11$ the minimal times in the $x$-only control case are even shorter than in the $x-y$ and $x-z$ cases. Since $x$-only control is easier to implement, this surprising observation provides an additional argument for using $x$-only control in the regime of interest for superconducting charge qubits.

As is well known 25 , the $\sqrt{\text { SWAP }}$ gate on two qubits is naturally implemented by the isotropic Heisenberg Hamiltonian after a time $\tau=\pi / 2 \approx 1.57$. As can be seen in Table 1 the minimal $\sqrt{\mathrm{SWAP}}_{3}$-gate times indeed seem to correspond to $\Delta \approx 1$ and are only slightly shorter than in the control-free case. This is despite the fact that our

Table 2. Minimal times (in units of $J^{-1}$ ) needed to reach fidelities higher than 0.999 for the relevant gates using only an $x$ control field.

\begin{tabular}{crc|rcc}
\hline$\Delta$ & \multicolumn{1}{c}{$\mathrm{X}_{3}$} & ${\sqrt{\text { SWAP }_{3}}}$ & \multicolumn{1}{c}{$\Delta$} & $\mathrm{X}_{3}$ & ${\sqrt{\mathrm{SWAP}_{3}}}^{1}$ \\
\hline 0.1 & 128.4 & 80.6 & 3.0 & 19.2 & 14.2 \\
0.2 & 70.4 & 45.0 & 4.0 & 18.4 & 9.5 \\
0.7 & 36.4 & 25.0 & 5.0 & 17.3 & 7.7 \\
0.8 & 37.1 & 25.6 & 6.0 & 16.4 & 7.5 \\
0.9 & 34.4 & 28.9 & 7.0 & 16.1 & 7.3 \\
1.0 & 18.8 & 25.8 & 8.0 & 15.5 & 6.4 \\
1.1 & 25.2 & 25.8 & 9.0 & 15.8 & 5.7 \\
1.2 & 31.1 & 31.9 & 10.0 & 15.8 & 3.9 \\
1.3 & 30.7 & 31.5 & 11.0 & 16.2 & 4.7 \\
2.0 & 23.2 & 21.7 & 12.0 & 16.5 & 4.3 \\
\hline
\end{tabular}

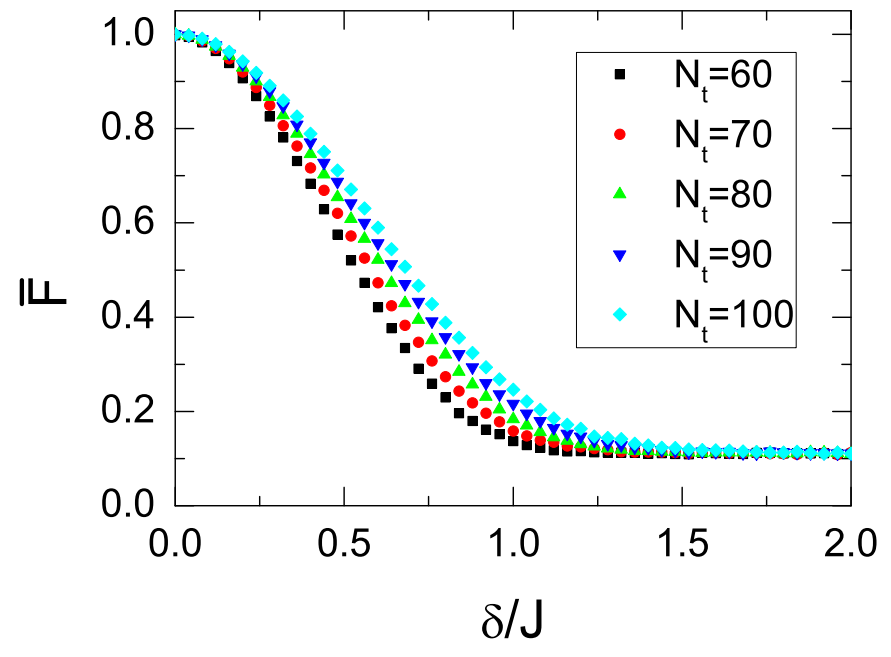

Fig. 2. (Color online) Average fidelity versus half-width $(\delta)$ for the $\sqrt{\mathrm{SWAP}}_{3}$ gate with $\Delta=1.2$ and fixed total time $t_{f}=60$.

$\sqrt{\mathrm{SWAP}}_{3}$ gate performs the $\sqrt{\mathrm{SWAP}}$ operation on the last two qubits (leaving the state of the first qubit unchanged) while the Heisenberg Hamiltonian of equation (2) also contains the interaction between the first two qubits. Thus we can conclude that the role of control fields in this case is to counteract the effect of the free evolution of the first qubit governed by $H_{0}$.

Generally speaking, the minimal gate times can in principle be found based on the time-optimal unitary operation formalism put forward by Carlini and co-workers 27 . This method requires solving a system of coupled nonlinear equations for Lagrange multipliers resulting from the quantum brachistochrone equation. In practice, extracting minimal times for different quantum gates in this way is feasible only when the time evolution of the total Hamiltonian of the system is as simple as to allow for an analytical solution of these equations. This is possible, for instance, when this Hamiltonian has a block-diagonal form in the computational basis, where each block commutes with itself at different times. In the problem at hand this is not the case, therefore an alternative strategy for finding minimal times is required.

\section{Robustness to random errors and finite pulse rise times}

In the following, we analyze the sensitivity of the fidelity to random errors in the control-field amplitudes, as well as to a finite rise time.

The random errors in control-field amplitudes are assumed to follow a uniform distribution of half-width $\delta$. For given $\delta$, we generate a large sample of $N \sim 1000$ control fields affected by random noise, for which we recalculate the fidelity. We are interested in the behavior of the average fidelity $\bar{F}=\sum_{i=1}^{N} F_{i} / N$, where the $F_{i}$ are fidelities for specific realizations of the random field, versus $\delta$ for the gates of interest and varying values of $\Delta$. 
In our previous work 16, using the isotropic Heisenberg model $(\Delta=1)$ as an example, it was demonstrated that the shape of the fidelity decay curves $(\bar{F}$ vs. $\delta)$ depends on the number of control pulses $N_{t}$ and their length $T$. Provided that the system satisfies the conditions for complete controllability, the saturation regime of the average fidelity sets in for $\delta \gtrsim 2 J$. The universal saturation value is $1 / d$, where $d$ is the dimension of the Hilbert space of the system. Importantly, for fixed $t_{f}=N_{t} T$, the average fidelity is closer to the intrinsic (in the absence of random errors) optimal values for larger $N_{t}$ (faster switching), this being a consequence of general properties of systems that exhibit competition between the resonanceand relaxation-type behavior [16]. Therefore, more rapid switching leads not only to higher intrinsic fidelities in the absence of randomness (recall section 4), but also renders these fidelities less sensitive to random errors. This is a manifestation of an intrinsic robustness of the system. Figure 2 illustrates that these features are also present in the anisotropic XXZ case.

The sensitivity to random errors in the control-field amplitudes depending on the anisotropy $\Delta$ is illustrated in Figure 3. As can be inferred from this figure, for larger $\Delta$ the system is less sensitive to random errors.

Another unavoidable source of imperfections in qubitarray realizations is the finite rise time of the control fields. Instead of a stepwise behavior, experimental control fields $h_{j, n}\left(j=x, y ; n=1, \ldots, N_{t} / 2\right)$ are expected to have a finite rise/decay time $\tau$. Figure 4 shows the dependence of the fidelity on the finite rise time. For larger values of $\Delta$, the fidelities of optimal control sequences seem to be less affected by the finite rise time.

The central result of this section is that values of $\Delta>1$ lead to both shorter gate times and a reduced sensitivity of the fidelity to random errors in control field and finite rise time. This provides a guiding principle for future implementations of qubit arrays.

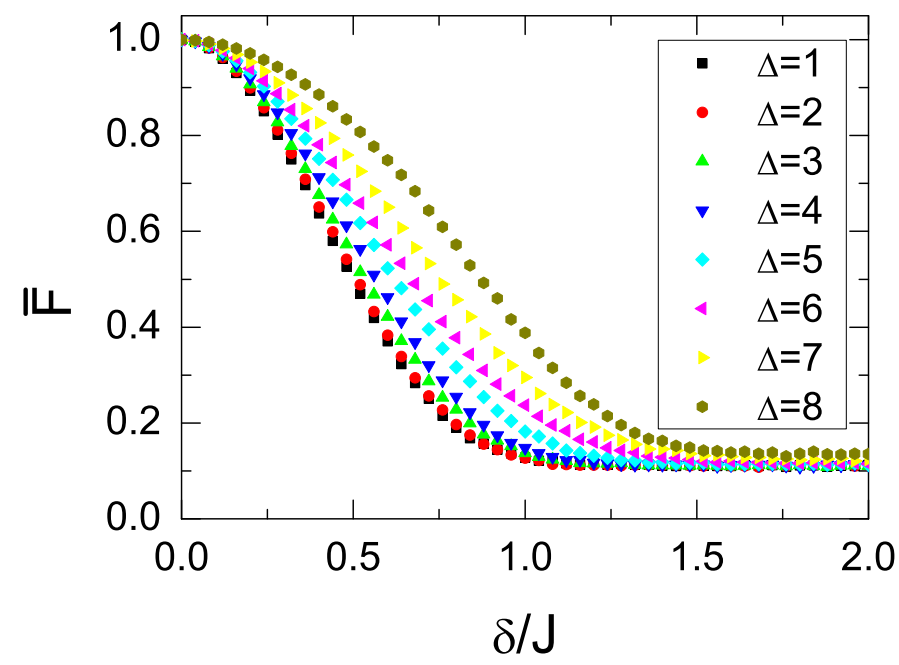

Fig. 3. (Color online) Average fidelity versus half-width $(\delta)$ of random-noise distribution for optimal control sequences with $N_{t}=70$ and $T=1$ corresponding to the $\mathrm{CNOT}_{3}$ gate.

\section{Discussion and Conclusions}

Our results are of direct relevance to superconducting qubit arrays [6]. One-dimensional Josephson arrays of capacitively coupled superconducting islands can be described as $X X Z$ Heisenberg spin-1/2 chains [28,29]. In general, the $X Y$-part of Hamiltonian is characterized by a nearestneighbor interaction, whereas the $Z$-part will also have coupling contributions beyond nearest neighbors. However, by properly choosing the junction capacitances and the capacitance of each island to the back gate of the structure, the $Z$-part will also be approximately of nearestneighbor type. The correspondence between the parameters of the Josephson array and the spin chain is as follows: the Josephson energy $E_{J}$ of the junctions coupling the islands corresponds to the exchange coupling constant $J$ of the spin system and can be controlled by a magnetic field if we assume that the coupling junctions are realized as SQUIDs. The parameter $\Delta J$ of the spin system corresponds to the charging energy $E_{C}$, i.e., the anisotropy parameter $\Delta$ corresponds to $E_{C} / E_{J}$. Values of $\Delta$ like those studied in Tables 1 and 2 can be experimentally realized. Finally, the first island should form a charge qubit, and the control field $h_{z}$ corresponds to the gate voltage, while $h_{x}$ and $h_{y}$ play the role of the Josephson energy of this charge qubit. Our study shows that, in principle, arbitrary quantum algorithms can be realized on a one-dimensional Josephson array by controlling only the first island in the array.

In summary, we have shown that local control of the first spin of an anisotropic $X X Z$ Heisenberg spin-1/2 chain enables universal quantum computation. Using a recent Lie-algebraic result on the local controllability of spin chains with "always-on" interactions, we have determined control pulses leading to optimal fidelities for quantum gates such as spin-flip (NOT), controlled-NOT (CNOT), and square-root-of-SWAP $(\sqrt{\mathrm{SWAP}})$. We have found the min-

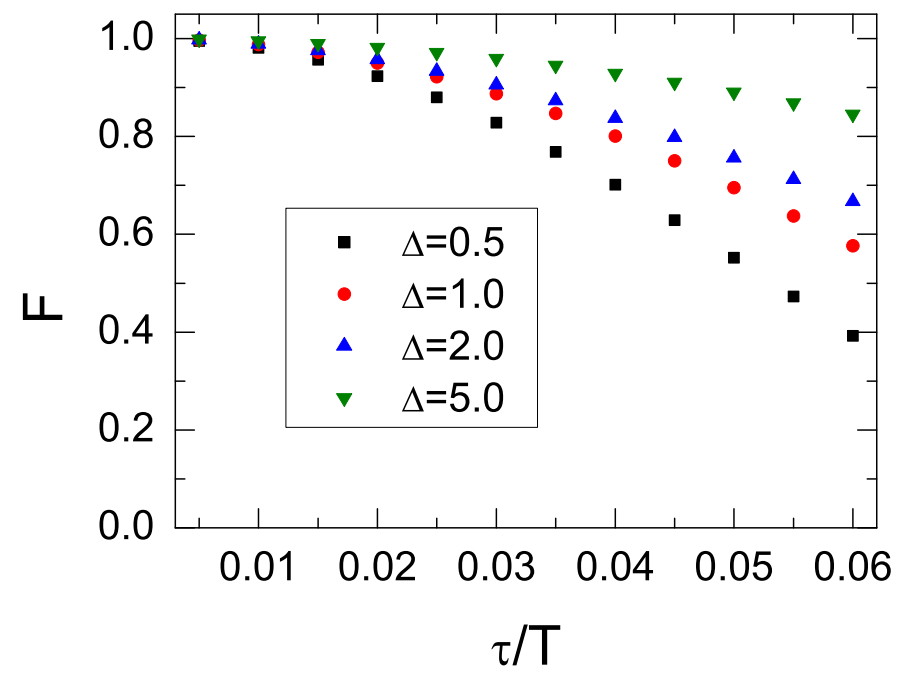

Fig. 4. (Color online) Illustration of sensitivity to finite rise time for the $X_{3}$ gate. The optimal control sequences used correspond to $N_{t}=70$ and $T=1$. 
imal times for realizing different gates depending on the anisotropy parameter $\Delta$ of the model, showing that the shortest among these gate times are achieved for particular values of $\Delta$ larger than unity. Another surprising result was that in the regime of interest for superconducting charge qubits, the minimal times in the simpler $x$-only control case can be even shorter than in the $x-y$ and $x$ $z$ control cases. We have also analyzed the sensitivity of the obtained results for the gate fidelities to random variations in the control-field amplitudes and finite rise time of the pulses. Our results are independent of a particular experimental realization of the $X X Z$ chain, yet, a superconducting Josephson array would be a particularly appealing candidate. Our investigation paves the way for future studies, involving more sophisticated control strategies 30,31 .

We would like to thank R. Fazio for discussions. This work was financially supported by EU project SOLID, the EPSRC grant EP/F043678/1, the Swiss NSF, and the NCCR Nanoscience.

\section{References}

1. S. Lloyd, A.J. Landahl, J.J.E. Slotine, Phys. Rev. A 69, 012305 (2004)

2. D. D'Alessandro, Introduction to Quantum Control and Dynamics (Taylor \& Francis, Boca Raton, 2008)

3. V. Jurdjevic, H.J. Sussmann, J. Differ. Equations 12, 313 (1972)

4. For a recent review, see C. Brif, R. Chakrabarti, H. Rabitz, New. J. Phys. 12, 075008 (2010).

5. See, e.g., S. Bose, Phys. Rev. Lett. 91, 207901 (2003)

6. A. Romito, R. Fazio, C. Bruder, Phys. Rev. B 71, 100501(R) (2005)

7. A.O. Lyakhov, C. Bruder, Phys. Rev. B 74, 235303 (2006)

8. D. Burgarth, Eur. Phys. J. Special Topics 151, 147 (2007)

9. T. Caneva, M. Murphy, T. Calarco, R. Fazio, S. Montangero, V. Giovannetti, G.E. Santoro, Phys. Rev. Lett. 103, 240501 (2009)

10. K. Maruyama, T. Iitaka, F. Nori, Phys. Rev. A 75, 012325 (2007)

11. S.G. Schirmer, I.C.H. Pullen, P.J. Pemberton-Ross, Phys. Rev. A 78, 062339 (2008)

12. D. Burgarth, S. Bose, C. Bruder, V. Giovannetti, Phys. Rev. A 79, 060305(R) (2009)

13. A. Kay, P.J. Pemberton-Ross, Phys. Rev. A 81, 010301(R) (2010)

14. D. Burgarth, K. Maruyama, M. Murphey, S. Montangero, T. Calarco, F. Nori, M.B. Plenio, Phys. Rev. A 81, 040303(R) (2010)

15. X. Wang, A. Bayat, S.G. Schirmer, S. Bose, Phys. Rev. A 81, $032312(2010)$

16. R. Heule, C. Bruder, D. Burgarth, V. M. Stojanović, arXiv:1007.2572 (2010)

17. Y. Makhlin, G. Schön, A. Shnirman, Rev. Mod. Phys. 73, $357(2001)$

18. L. S. Levitov, T. P. Orlando, J. B. Majer, J. E. Mooij, arXiv:cond-mat/0108266v2 (2001)

19. J.Q. You, F. Nori, Phys. Today 58, 42 (2005)

20. T. Giamarchi, Quantum Physics in One Dimension (Clarendon Press, Oxford, 2004)
21. S.G. Schirmer, H. Fu, A.I. Solomon, Phys. Rev. A 63, 063410 (2001)

22. W. Pfeifer, The Lie Algebras $s u(N)$ : An Introduction (Birkhäuser, Basel, 2003)

23. T. Polack, H. Suchowski, D.J. Tannor, Phys. Rev. A 79, 053403 (2009)

24. U. Sander, T. Schulte-Herbrüggen, arXiv:0904.4654

25. G. Burkard, D. Loss, D. P. Di Vincenzo, J. A. Smolin, Phys. Rev. B 60, 11404 (1999)

26. W.H. Press, S.A. Teukolsky, W.T. Vetterling, B.P. Flannery, Numerical Recipes in Fortran 77 and 90: The Art of Scientific and Parallel Computing (Cambridge University Press, Cambridge, 1997)

27. A. Carlini, A. Hosoya, T. Koike, Y. Okudaira, Phys. Rev. A 75, 042308 (2007)

28. C. Bruder, R. Fazio, G. Schön, Phys. Rev. B 47, 342 (1993)

29. R. Fazio, H. van der Zant, Physics Reports 355, 235 (2001)

30. Y. Makhlin, Quant. Info. Proc. 1, 243 (2002)

31. S. Montangero, T. Calarco, R. Fazio, Phys. Rev. Lett. 99, 170501 (2007) 